\title{
Homoclinic solutions for a class of prescribed mean curvature Liénard equations
}

Shiping Lu*

\section{"Correspondence:}

lushiping88@sohu.com

College of Mathematics and

Statistics, Nanjing University of

Information Science and

Technology, Nanjing, 210044, China

\begin{abstract}
In this paper, the problem of a homoclinic solution is studied for the prescribed mean curvature Liénard equation $\left(\frac{u^{\prime}(t)}{\sqrt{1+\left(u^{\prime}(t)\right)^{2}}}\right)^{\prime}+f(u(t)) u^{\prime}(t)+g(u(t))=p(t)$, where $f \in C(R, R)$, $g \in C^{1}(R, R)$, and $p \in C(R, R)$. Under some conditions, the author obtains the result that the equation has at least one nontrivial homoclinic solution for $p(t) \not \equiv 0$, and the equation has no nontrivial homoclinic solution for $p(t) \equiv 0$. The arguments are based upon Mawhin's continuation theorem.
\end{abstract}

Keywords: homoclinic solution; periodic solution; Mawhin's continuation theorem; prescribed mean curvature equation

\section{Introduction}

In this paper, we investigate the existence and non-existence of nontrivial homoclinic solutions for a class of prescribed mean curvature equations,

$$
\left(\frac{u^{\prime}(t)}{\sqrt{1+\left(u^{\prime}(t)\right)^{2}}}\right)^{\prime}+f(u(t)) u^{\prime}(t)+g(u(t))=p(t)
$$

where $f \in C(R, R), g \in C^{1}(R, R), p \in C(R, R)$.

As is well known, a solution $u(t)$ of (1.1) is named homoclinic (to 0$)$ if $u(t) \rightarrow 0$ and $u^{\prime}(t) \rightarrow 0$ as $|t| \rightarrow+\infty$. In addition, if $u \neq 0$, then $u$ is called a nontrivial homoclinic solution.

A prescribed mean curvature equation arises from some problems associated with differential geometry and physics (see [1-8] and the references therein). In the past years, the problem of periodic solutions for the prescribed mean curvature equation has attracted many researchers' attention [9-13]. For example, by using an approach based on the LeraySchauder degree, the authors in [10] studied the periodic solutions for nonlinear equations with mean curvature-like operators. Considering the delay phenomenon to exist generally in nature, Feng in [11] studied the existence of periodic solutions for a prescribed mean curvature Liénard equation with delay as follows:

$$
\left(\frac{u^{\prime}(t)}{\sqrt{1+\left(u^{\prime}(t)\right)^{2}}}\right)^{\prime}+f(u(t)) u^{\prime}(t)+g(t, u(t-\tau(t)))=e(t)
$$

(c) 2015 Lu. This article is distributed under the terms of the Creative Commons Attribution 4.0 International License (http://creativecommons.org/licenses/by/4.0/), which permits unrestricted use, distribution, and reproduction in any medium, provided you give appropriate credit to the original author(s) and the source, provide a link to the Creative Commons license, and indicate if changes were made. 
S Lu and M Lu in [12] further studied the existence and non-existence of periodic solutions for the following prescribed mean curvature equation with multiple delays:

$$
\frac{d}{d t}\left(\frac{x^{\prime}(t)}{\sqrt{1+\left(x^{\prime}(t)\right)^{2}}}\right)+\sum_{i=1}^{n} a_{i}(t) g\left(x\left(t-\tau_{i}(t)\right)\right)=p(t)
$$

and Li in [13] studied the existence of periodic solutions for a prescribed mean curvature Rayleigh equation. Recently, the problem of existence of homoclinic solutions for some second-order Hamiltonian systems has been extensively studied by using critical point theory [14-17]. However, the problem of homoclinic solution has been rarely studied for a prescribed mean curvature equation like (1.1). Liang and Lu in [18] investigated the existence of a homoclinic solution for the following equation:

$$
\left(\frac{u^{\prime}(t)}{\sqrt{1+\left(u^{\prime}(t)\right)^{2}}}\right)^{\prime}+c u^{\prime}(t)+f(u(t))=p(t)
$$

where $c>0$ is a constant, but the term containing the first derivative is only linear with respect to $u^{\prime}(t)$.

Let $T$ be a positive constant, like in the work of $[18,19]$, for each $k \in \mathbf{N}$, we investigate the existence of $2 k T$-periodic solutions $u_{k}(t)$ for the following equation:

$$
\left(\frac{u^{\prime}(t)}{\sqrt{1+\left(u^{\prime}(t)\right)^{2}}}\right)^{\prime}+f(u(t)) u^{\prime}(t)+g(u(t))=p_{k}(t)
$$

where $p_{k}: R \rightarrow R$ is a $2 k T$-periodic function such that

$$
p_{k}(t)= \begin{cases}p(t), & t \in[-k T, k T-\varepsilon), \\ p(k T-\varepsilon)+\frac{p(-k T)-p(k T-\varepsilon)}{\varepsilon}(t-k T+\varepsilon), & t \in[k T-\varepsilon, k T],\end{cases}
$$

$\varepsilon \in\left(0, T_{0}\right)$ is a constant independent of $k, T_{0}=\min \{T, 1\}$. Then a homoclinic solution for (1.1) is obtained as a limit of a certain sequence of $\left\{u_{k}(t)\right\}$.

In this paper, unlike the methods based on critical point theory for guaranteeing the existence of homoclinic solutions in the work of [14-17], our approach for obtaining the existence of homoclinic solutions for (1.1) is based on topological degree theory. In detail, the existence of $2 k T$-periodic solutions to (1.4) is obtained by using Mawhin's continuation theorem [20], not by using critical point theory. This is due to the fact that there is a first derivative term $f(u(t)) u^{\prime}(t)$ in (1.4), and then (1.4) is not the Euler-Lagrange equation associated with some functional. Furthermore, we not only investigate the existence of a homoclinic solution to (1.1), but we also study the non-existence of a homoclinic solution to $(1.1)$.

\section{Preliminary}

In order to use Mawhin's continuation theorem [20], we first recall it.

Let $X$ and $Y$ be two Banach spaces with norms $\|\cdot\|_{X},\|\cdot\|_{Y}$, respectively. A linear operator $L: D(L) \subset X \rightarrow Y$ is said to be a Fredholm operator of index zero provided that:

(a) $\operatorname{Im} L$ is a closed subset of $Y$,

(b) $\operatorname{dim} \operatorname{Ker} L=\operatorname{codim} \operatorname{Im} L<\infty$.

Let $X$ and $Y$ be two Banach spaces with norms $\|\cdot\|_{X},\|\cdot\|_{Y}$, respectively, $\Omega \subset X$ be an open and bounded set and $L: D(L) \subset X \rightarrow Y$ be a Fredholm operator of index zero. The 
continuous operator $N: \Omega \subset X \rightarrow Y$ is said to be $L$-compact in $\bar{\Omega}$ provided that:

(c) $K_{p}(I-Q) N(\bar{\Omega})$ is a relative compact set of $X$,

(d) $Q N(\bar{\Omega})$ is a bounded set of $Y$,

and where we denote $X_{1}=\operatorname{Ker} L, Y_{2}=\operatorname{Im} L$ we have the decompositions $X=X_{1} \oplus X_{2}, Y=$ $Y_{1} \oplus Y_{2}$. Let $P: X \rightarrow X_{1}, Q: Y \rightarrow Y_{1}$ be continuous linear projectors (meaning $P^{2}=P$ and $\left.Q^{2}=Q\right)$, and $K_{p}=\left.L\right|_{\mathrm{Ker} P \cap D(L)} ^{-1}$.

Lemma 2.1 ([20]) Let $X$ and $Y$ be two Banach spaces with norms $\|\cdot\|_{X},\|\cdot\|_{Y}$, respectively, $\Omega$ be an open and bounded set of $X$, and $L: D(L) \subset X \rightarrow Y$ be a Fredholm operator of index zero. The operator $N: \bar{\Omega} \subset X \rightarrow Y$ is said to be L-compact in $\bar{\Omega}$. In addition, if the following conditions hold:

(H1) $L v \neq \lambda N v, \forall(v, \lambda) \in \partial \Omega \times(0,1)$;

(H2) $Q N v \neq 0, \forall v \in \operatorname{Ker} L \cap \partial \Omega$;

(H3) $\operatorname{deg}\{J Q N, \Omega \cap \operatorname{Ker} L, 0\} \neq 0$, where $J: \operatorname{Im} Q \rightarrow \operatorname{Ker} L$ is a homeomorphism, then $L v=N v$ has at least one solution in $D(L) \cap \bar{\Omega}$.

Consider the following system:

$$
\left\{\begin{array}{l}
x^{\prime}(t)=\varphi(y(t))=\frac{y(t)}{\sqrt{1-y^{2}(t)}}, \\
y^{\prime}(t)=f(x(t)) \varphi(y(t))-g(x(t))+p(t) .
\end{array}\right.
$$

Obviously, $(x(t), y(t))^{\top}$ is a solution of (2.1), then $x(t)$ must be a solution of (1.1), and finding homoclinic solutions of (1.1) is equivalent to finding a solution $(x(t), y(t))^{\top}$ of (2.1) such that $(x(t), y(t)) \rightarrow(0,0)$ as $|t| \rightarrow+\infty$. Similarly, finding a $2 k T$-periodic solution to (1.4) is equivalent to finding a $2 k T$-periodic solution to the system

$$
\left\{\begin{array}{l}
x^{\prime}(t)=\varphi(y(t))=\frac{y(t)}{\sqrt{1-y^{2}(t)}} \\
y^{\prime}(t)=-f(x(t)) \varphi(y(t))-g(x(t))+p_{k}(t),
\end{array}\right.
$$

where $y(t)=\frac{x^{\prime}(t)}{\sqrt{1+\left(x^{\prime}(t)\right)^{2}}}$. It is easy to see that $\max _{t \in[-k T, k T]}|y(t)|<1$. Let $X_{k}=Y_{k}=\{v=$ $\left.(x, y)^{\top} \in C\left(R, R^{2}\right), v(t)=v(t+2 k T)\right\}$, where the normal is defined by $\|v\|=\max \left\{|x|_{0},|y|_{0}\right\}$, where $|x|_{0}=\max _{t \in[0,2 k T]}|x(t)|,|y|_{0}=\max _{t \in[0,2 k T]}|y(t)|$. It is obvious that $X_{k}$ and $Y_{k}$ are Banach spaces.

Furthermore, for $x \in X_{k}$, denote $\|x\|_{p}$ by $\|x\|_{p}=\left(\int_{-k T}^{k T}|x(t)|^{p} d t\right)^{1 / p}$, where $p \geq 1$ is a constant.

Now we define the operator

$$
L: D(L) \subset X_{k} \rightarrow Y_{k}, \quad L v=v^{\prime}=\left(x^{\prime}(t), y^{\prime}(t)\right)^{\top},
$$

where $D(L)=\left\{v \mid v=(x, y)^{\top} \in C^{1}\left(R, R^{2}\right), v(t)=v(t+2 k T)\right\}$.

Let $Z_{k}=\left\{v \mid v=(x, y)^{\top} \in C(R, R \times(-1,1)), v(t)=v(t+2 k T)\right\}$. Define a nonlinear operator $N: \bar{\Omega} \subset Z_{k} \subset X_{k} \rightarrow Y_{k}$,

$$
N v=\left(\frac{y(t)}{\sqrt{1-y^{2}(t)}},-f(x(t)) \varphi(y(t))-g(x(t))+p_{k}(t)\right)^{\top} .
$$

Then problem (2.2) can be written as $L v=N v$ in $\bar{\Omega}$. 
Clearly, $\operatorname{Ker} L=\left\{v \mid v \in X_{k}, v^{\prime}=\left(x^{\prime}(t), y^{\prime}(t)\right)^{\top}=(0,0)^{\top}\right\}=R^{2}$, and it is also easy to prove that $\operatorname{Im} L=\left\{z \in Y_{k}, \int_{0}^{2 k T} z(s) d s=0\right\}$, so $L$ is a Fredholm operator of index zero.

Let $P: X_{k} \rightarrow \operatorname{Ker} L, P v=\frac{1}{2 k T} \int_{0}^{2 k T} v(s) d s, Q: Y_{k} \rightarrow \operatorname{Im} Q, Q z=\frac{1}{2 k T} \int_{0}^{2 k T} z(s) d s$. If set $K_{p}=$ $\left.L\right|_{\operatorname{Ker} p \cap D(L)} ^{-1}$, then

$$
\left(K_{p} z\right)(t)=\int_{0}^{2 k T} G(t, s) z(s) d s
$$

where

$$
G(t, s)= \begin{cases}\frac{s-2 k T}{2 k T}, & 0 \leq t \leq s \\ \frac{s}{2 k T}, & s \leq t \leq 2 k T\end{cases}
$$

For all $\Omega$ such that $\bar{\Omega} \subset Z_{k} \subset X_{k}$, we have $K_{p}(I-Q) N(\bar{\Omega})$ is a relative compact set of $X_{k}$, $Q N(\bar{\Omega})$ is a bounded set of $Y_{k}$, so the operator $N$ is $L$-compact in $\bar{\Omega}$.

Remark 2.1 In view of the definition of the nonlinear operator $N: \bar{\Omega} \subset Z_{k} \subset X_{k} \rightarrow Y_{k}$ in (2.3), we see that there must be two points $\rho_{0} \in(0,+\infty)$ and $\rho_{1} \in(0,1)$ such that the open and bounded set $\Omega$, which is associated with Lemma 2.1, should be $\Omega=\left\{v \mid v=(x, y)^{\top} \in\right.$ $\left.Z_{k},|x|_{0}<\rho_{0},|y|_{0}<\rho_{1}\right\}$.

Lemma 2.2 If $u: R \rightarrow R$ is continuously differentiable on $R, a>0, \mu>1$, and $p>1$ are constants, then for every $t \in R$, the following inequality holds:

$$
|u(t)| \leq(2 a)^{-\frac{1}{\mu}}\left(\int_{t-a}^{t+a}|u(s)|^{\mu} d s\right)^{\frac{1}{\mu}}+a(2 a)^{-\frac{1}{p}}\left(\int_{t-a}^{t+a}\left|u^{\prime}(s)\right|^{p} d s\right)^{\frac{1}{p}} .
$$

This lemma is a special case of Lemma 2.2 in [16].

Lemma 2.3 ([21]) Let $u_{k}:=\left(x_{k}, y_{k}\right)^{\top} \in C_{2 k T}^{1}$ be a $2 k T$-periodic function, such that for each $k \in \mathbf{N},\left(x_{k}, y_{k}\right)$ satisfies

$$
\left|x_{k}\right|_{0} \leq A_{0}, \quad\left|x_{k}^{\prime}\right|_{0} \leq A_{1}, \quad\left|y_{k}\right|_{0} \leq B_{0}, \quad\left|y_{k}^{\prime}\right|_{0} \leq B_{1}
$$

where $A_{0}, A_{1}, B_{1}$, and $B_{2}$ are constants independent of $k \in \mathbf{N}$. Then there exist a $u_{0} \in$ $C\left(R, R^{2}\right)$ and a subsequence $\left\{u_{k_{j}}\right\}$ of $\left\{u_{k}\right\}_{k \in \mathbf{N}}$ such that for each $j \in \mathbf{N}$,

$$
\max _{t \in[-j T, j T]}\left|u_{k_{i}}(t)-u_{0}(t)\right| \rightarrow 0 \quad \text { as } i \rightarrow+\infty
$$

\section{Main results}

For the sake of convenience, we list the following assumptions:

$\left(\mathrm{A}_{1}\right)$ There exist constants $m_{0}>0, l>0$, and $m>1$ such that $x g(x) \leq-m_{0}|x|^{m},|f(x)| \geq l$, and $g^{\prime}(x) \leq 0, \forall x \in R$.

$\left(\mathrm{A}_{2}\right) p \in C(R, R)$ with $p(t) \not \equiv 0$ and $\sup _{t \in R}|p(t)|<+\infty ; \int_{R}|p(t)|^{\frac{m}{m-1}} d t<+\infty$ and $\int_{R}|p(t)|^{2} d t<+\infty$, where $m$ is determined in assumption $\left(\mathrm{A}_{1}\right)$. 
Remark 3.1 Let

$$
|p|_{\infty}:=\sup _{t \in R}|p(t)|, \quad \alpha:=\left(\int_{R}|p(t)|^{\frac{m}{m-1}} d t\right)^{\frac{m-1}{m}}, \quad \beta:=\left(\int_{R}|p(t)|^{2} d t\right)^{\frac{1}{2}} .
$$

From (1.4), and by calculating directly, we can obtain the following result.

Lemma 3.1 If assumption $\left(\mathrm{A}_{2}\right)$ holds, then $\alpha<+\infty, \beta<+\infty$; and for all $k \in \mathbf{N}$,

$$
\begin{aligned}
& \left|p_{k}\right|_{0} \leq|p|_{\infty}, \quad\left\|p_{k}\right\|_{\frac{m}{m-1}}:=\left(\int_{-k T}^{k T}\left|p_{k}(t)\right|^{\frac{m}{m-1}} d t\right)^{\frac{m-1}{m}} \leq \alpha+\varepsilon^{\frac{m-1}{m}}|p|_{\infty} \\
& \left\|p_{k}\right\|_{2}:=\left(\int_{-k T}^{k T}\left|p_{k}(t)\right|^{2} d t\right)^{\frac{1}{2}} \leq \beta+\varepsilon^{1 / 2}|p|_{\infty} .
\end{aligned}
$$

In order to study the existence of $2 k T$-periodic solutions to (2.2), we firstly study some properties of all possible $2 k T$-periodic solutions $\left(x_{k}, y_{k}\right)^{\top}$ to the following system:

$$
\left\{\begin{array}{l}
x^{\prime}(t)=\lambda \varphi(y(t))=\lambda \frac{y(t)}{\sqrt{1-y^{2}(t)}}, \\
y^{\prime}(t)=-\lambda f(x(t)) \varphi(y(t))-\lambda g(x(t))+\lambda p_{k}(t), \quad \lambda \in(0,1] .
\end{array}\right.
$$

Obviously, $\left(x_{k}, y_{k}\right)^{\top} \in Z_{k} \subset X_{k}$. For each $k \in \mathbf{N}$, let $\Sigma_{k}$ represent the set of all the $2 k T$ periodic solutions to the above system. This is

$$
\begin{aligned}
\Sigma_{k}:= & \left\{(x, y) \in Z_{k} \mid x^{\prime}(t)=\frac{\lambda y(t)}{\sqrt{1-y^{2}(t)}},\right. \\
& \left.y^{\prime}(t)=-\lambda f(x(t)) \varphi(y(t))-\lambda g(x(t))+\lambda p_{k}(t), \lambda \in(0,1]\right\} .
\end{aligned}
$$

Theorem 3.1 Assume that conditions $\left(\mathrm{A}_{1}\right)$ and $\left(\mathrm{A}_{2}\right)$ hold, and the constant $T$ satisfies

$$
\rho_{1}:=(2 T)^{-\frac{1}{2}}\left(\frac{\alpha^{m}}{m_{0}}\right)^{\frac{1}{2(m-1)}}+\beta \sqrt{\frac{T}{2}}\left[\frac{M}{l}+1\right]<1
$$

where $M=\sup _{|x| \leq M_{0}}|f(x)|$,

$$
M_{0}=(2 T)^{\frac{1}{m}}\left(\frac{\alpha}{m_{0}}\right)^{\frac{1}{m-1}}+\sqrt{\frac{T}{2}} \frac{\beta}{l}
$$

For each $k \in \mathbf{N}$, if $(x, y)^{\top} \in \Sigma_{k}$, there are positive constants $A_{0}, A_{1}, B_{0}, B_{1}, \rho_{0}, \rho_{2}$, and $\rho_{3}$, which are all independent of $k$ and $\lambda$, such that

$$
\|x\|_{m} \leq A_{0}, \quad\left\|x^{\prime}\right\|_{2} \leq A_{1}, \quad\|y\|_{2} \leq B_{0}, \quad\left\|y^{\prime}\right\|_{2} \leq B_{1}
$$

and

$$
|x|_{0} \leq \rho_{0}, \quad|y|_{0} \leq \rho_{1}<1, \quad\left|x^{\prime}\right|_{0} \leq \rho_{2}, \quad\left|y^{\prime}\right|_{0} \leq \rho_{3} .
$$


Proof For each $k \in \mathbf{N}$, if $(x, y)^{\top} \in \Sigma_{k}$, then

$$
\left\{\begin{array}{l}
x^{\prime}(t)=\lambda \varphi(y(t))=\lambda \frac{y(t)}{\sqrt{1-y^{2}(t)}}, \\
y^{\prime}(t)=-\lambda f(x(t)) \varphi(y(t))-\lambda g(x(t))+\lambda p_{k}(t), \quad \lambda \in(0,1] .
\end{array}\right.
$$

Multiplying the first equation of (3.4) by $y^{\prime}(t)$ and integrating from $-k T$ to $k T$, we have

$$
\int_{-k T}^{k T} y^{\prime}(t) x^{\prime}(t) d t=\int_{-k T}^{k T} y^{\prime}(t) \lambda \varphi(y(t)) d t=\int_{-k T}^{k T} \lambda \varphi(y(t)) d y(t)=0 .
$$

Multiplying the second equation of (3.4) by $x(t)$ and integrating from $-k T$ to $k T$, we have

$$
\begin{aligned}
& \int_{-k T}^{k T} y^{\prime}(t) x(t) d t \\
& \quad=-\int_{-k T}^{k T} y(t) x^{\prime}(t) d t=-\lambda \int_{-k T}^{k T} \frac{y^{2}(t)}{\sqrt{1-y^{2}(t)}} d t \\
& \quad=\lambda\left(-\int_{-k T}^{k T} x(t) f(x(t)) \varphi(y(t)) d t-\int_{-k T}^{k T} x(t) g(x(t)) d t+\int_{-k T}^{k T} x(t) p_{k}(t) d t\right),
\end{aligned}
$$

i.e.,

$$
\begin{aligned}
& \int_{-k T}^{k T} \frac{y^{2}(t)}{\sqrt{1-y^{2}(t)}} d t-\int_{-k T}^{k T} x(t) g(x(t)) d t-\int_{-k T}^{k T} x(t) f(x(t)) \varphi(y(t)) d t \\
& \quad=-\int_{-k T}^{k T} x(t) p_{k}(t) d t \\
& \quad \leq \int_{-k T}^{k T}|x(t)|\left|p_{k}(t)\right| d t .
\end{aligned}
$$

From the first equation of (3.4), we have

$$
\int_{-k T}^{k T} x(t) f(x(t)) \varphi(y(t)) d t=\lambda^{-1} \int_{-k T}^{k T} x(t) f(x(t)) x^{\prime}(t) d t=0,
$$

which together (3.6) with $\left(A_{1}\right)$ and $\left(A_{2}\right)$ yields

$$
\|y\|_{2}^{2}+m_{0}\|x\|_{m}^{m} \leq \int_{-k T}^{k T}|x(t)|\left|p_{k}(t)\right| d t .
$$

Applying Hölder's inequality to the above inequality, we obtain

$$
\|y\|_{2}^{2}+m_{0}\|x\|_{m}^{m} \leq\left\|p_{k}\right\|_{\frac{m}{m-1}}\|x\|_{m}
$$

which implies that

$$
m_{0}\|x\|_{m}^{m} \leq\left\|p_{k}\right\|_{\frac{m}{m-1}}\|x\|_{m}
$$

and

$$
\|y\|_{2}^{2} \leq\left\|p_{k}\right\|_{\frac{m}{m-1}}\|x\|_{m} .
$$


It follows from (3.7) and (3.8) that

$$
\|x\|_{m} \leq\left(\frac{\left\|p_{k}\right\|_{\frac{m}{m-1}}}{m_{0}}\right)^{\frac{1}{m-1}} \leq\left(\frac{\alpha+\varepsilon^{\frac{m-1}{m}}|p|_{\infty}}{m_{0}}\right)^{\frac{1}{m-1}}:=A_{0}(\varepsilon)
$$

and

$$
\|y\|_{2}<\left(\frac{\left(\alpha+\varepsilon^{\frac{m-1}{m}}|p|_{\infty}\right)^{m}}{m_{0}}\right)^{\frac{1}{2(m-1)}}:=B_{0}(\varepsilon)
$$

Multiplying the second equation of (3.4) by $x^{\prime}(t)$ and integrating from $-k T$ to $k T$, we have

$$
\begin{aligned}
\int_{-k T}^{k T} y^{\prime}(t) x^{\prime}(t) d t= & -\lambda \int_{-k T}^{k T} f(x(t))\left(x^{\prime}(t)\right)^{2} d t-\lambda \int_{-k T}^{k T} g(x(t)) x^{\prime}(t) d t \\
& +\lambda \int_{-k T}^{k T} x^{\prime}(t) p_{k}(t) d t .
\end{aligned}
$$

It follows from (3.5) and the condition $|f(x)| \geq l$ for all $x \in R$ that

$$
\begin{aligned}
l \int_{-k T}^{k T}\left|x^{\prime}(t)\right|^{2} d t & \leq \int_{-k T}^{k T}\left|x^{\prime}(t)\right|\left|p_{k}(t)\right| d t \\
& \leq\left\|x^{\prime}\right\|_{2}\left\|p_{k}\right\|_{2},
\end{aligned}
$$

i.e.,

$$
\left\|x^{\prime}\right\|_{2}=\left(\int_{-k T}^{k T}\left|x^{\prime}(t)\right|^{2} d t\right)^{1 / 2} \leq \frac{\left\|p_{k}\right\|_{2}}{l} \leq \frac{\beta+\varepsilon^{1 / 2}|p|_{\infty}}{l}:=A_{1}(\varepsilon) .
$$

From (3.9), and by applying Lemma 2.2, we have

$$
\begin{aligned}
|x|_{0} & \leq(2 T)^{1 / m}\left(\int_{t-T}^{t+T}|x(s)|^{m} d t\right)^{1 / m}+T(2 T)^{-\frac{1}{2}}\left(\int_{t-T}^{t+T}\left|x^{\prime}(s)\right|^{2} d t\right)^{1 / 2} \\
& \leq(2 T)^{1 / m}\left(\int_{t-k T}^{t+k T}|x(s)|^{m} d t\right)^{1 / m}+T(2 T)^{-\frac{1}{2}}\left(\int_{t-k T}^{t+k T}\left|x^{\prime}(s)\right|^{2} d t\right)^{1 / 2} \\
& \leq(2 T)^{1 / m}\left(\int_{-k T}^{k T}|x(s)|^{m} d t\right)^{1 / m}+T(2 T)^{-\frac{1}{2}}\left(\int_{-k T}^{k T}\left|x^{\prime}(s)\right|^{2} d t\right)^{1 / 2} \\
& \leq(2 T)^{1 / m} A_{0}(\varepsilon)+T(2 T)^{-\frac{1}{2}} A_{1}(\varepsilon) \\
& :=\rho_{0}(\varepsilon) .
\end{aligned}
$$

Multiplying the second equation of (3.4) by $y^{\prime}(t)$ and integrating from $-k T$ to $k T$, we have

$$
\begin{aligned}
& \int_{-k T}^{k T}\left(y^{\prime}(t)\right)^{2} d t \\
& \quad=-\int_{-k T}^{k T} \lambda y^{\prime}(t) g(x(t)) d t-\int_{-k T}^{k T} \lambda y^{\prime}(t) f(x(t)) \varphi(y(t)) d t+\int_{-k T}^{k T} \lambda y^{\prime}(t) p_{k}(t) d t
\end{aligned}
$$




$$
\begin{aligned}
= & \int_{-k T}^{k T} \lambda^{2} g^{\prime}(x(t)) \frac{y^{2}(t)}{\sqrt{1-y^{2}(t)}} d t-\int_{-k T}^{k T} \lambda y^{\prime}(t) f(x(t)) \varphi(y(t)) d t \\
& +\int_{-k T}^{k T} \lambda y^{\prime}(t) p_{k}(t) d t .
\end{aligned}
$$

Since

$$
\lambda \int_{-k T}^{k T} y^{\prime}(t) f(x(t)) \varphi(y(t)) d t=\int_{-k T}^{k T} x^{\prime}(t) y^{\prime}(t) f(x(t)) d t
$$

it follows from (3.12) that

$$
\left|\int_{-k T}^{k T} y^{\prime}(t) f(x(t)) \varphi(y(t)) d t\right| \leq f_{\rho_{0}(\varepsilon)}\left\|x^{\prime}\right\|_{2}\left\|y^{\prime}\right\|_{2}
$$

which together with (3.11) gives

$$
\left|\int_{-k T}^{k T} y^{\prime}(t) f(x(t)) \varphi(y(t)) d t\right| \leq f_{\rho_{0}(\varepsilon)} A_{1}(\varepsilon)\left\|y^{\prime}\right\|_{2} .
$$

Moreover, by using assumption $\left(A_{1}\right)$, we see

$$
\int_{-k T}^{k T} \lambda^{2} g^{\prime}(x(t)) \frac{y^{2}(t)}{\sqrt{1-y^{2}(t)}} d t \leq 0 .
$$

Substituting it and (3.14) into (3.13), we have

$$
\int_{-k T}^{k T}\left(y^{\prime}(t)\right)^{2} d t \leq f_{\rho_{0}(\varepsilon)} A_{1}(\varepsilon)\left\|y^{\prime}\right\|_{2}+\left\|p_{k}\right\|_{2}\left\|y^{\prime}\right\|_{2},
$$

i.e.,

$$
\left\|y^{\prime}\right\|_{2} \leq f_{\rho_{0}(\varepsilon)} A_{1}(\varepsilon)+\left\|p_{k}\right\|_{2} \leq f_{\rho_{0}(\varepsilon)} A_{1}(\varepsilon)+\beta+\varepsilon^{1 / 2}|p|_{\infty}:=B_{1}(\varepsilon) .
$$

Thus by using Lemma 2.2 , for all $t \in[-k T, k T]$, we get

$$
\begin{aligned}
|y(t)| & \leq(2 T)^{-\frac{1}{2}}\left(\int_{t-T}^{t+T}|y(s)|^{2} d s\right)^{\frac{1}{2}}+T(2 T)^{-\frac{1}{2}}\left(\int_{t-T}^{t+T}\left|y^{\prime}(s)\right|^{2} d s\right)^{\frac{1}{2}} \\
& \leq(2 T)^{-\frac{1}{2}}\left(\int_{t-k T}^{t+k T}|y(s)|^{2} d s\right)^{\frac{1}{2}}+T(2 T)^{-\frac{1}{2}}\left(\int_{t-k T}^{t+k T}\left|y^{\prime}(s)\right|^{2} d s\right)^{\frac{1}{2}} \\
& =(2 T)^{-\frac{1}{2}}\left(\int_{-k T}^{k T}|y(s)|^{2} d s\right)^{\frac{1}{2}}+T(2 T)^{-\frac{1}{2}}\left(\int_{-k T}^{k T}\left|y^{\prime}(s)\right|^{2} d s\right)^{\frac{1}{2}},
\end{aligned}
$$

and then by (3.9) and (3.11), we have

$$
\begin{aligned}
|y|_{0} & =\max _{t \in[-k T, k T]}|y(t)| \leq(2 T)^{-\frac{1}{2}} B_{0}(\varepsilon)+\sqrt{\frac{T}{2}} B_{1}(\varepsilon) \\
& =(2 T)^{-\frac{1}{2}}\left(\frac{\left(\alpha+\varepsilon^{\frac{m-1}{m}}|p|_{\infty}\right)^{m}}{m_{0}}\right)^{\frac{1}{2(m-1)}}+\sqrt{\frac{T}{2}}\left[f_{\rho_{0}(\varepsilon)} A_{1}(\varepsilon)+\beta+\varepsilon^{1 / 2}|p|_{\infty}\right] \\
& :=\rho(\varepsilon) .
\end{aligned}
$$


From (3.9), (3.10), (3.11), and (3.12), we see

$$
\lim _{\varepsilon \rightarrow 0} \rho(\varepsilon)=\rho(0)=(2 T)^{-\frac{1}{2}}\left(\frac{\alpha^{m}}{m_{0}}\right)^{\frac{1}{2(m-1)}}+\beta \sqrt{\frac{T}{2}}\left[\frac{M}{l}+1\right],
$$

where $M=\sup _{|x| \leq \rho_{0}(0)}|f(x)|$ is a constant determined in (3.1). It follows from (3.1) that there is a constant $\delta>0$ such that

$$
\rho(\varepsilon)<1, \quad \forall \varepsilon \in(0, \delta]
$$

This implies that if the constant $\varepsilon$ determined in (1.5) is chosen as in $(0, \delta]$, then it follows from (3.16) that

$$
|y|_{0} \leq \rho_{1}:=\rho(\varepsilon)<1
$$

and then by the first equation of (3.4), we get

$$
\left|x^{\prime}\right|_{0}<\frac{\rho_{1}}{\sqrt{1-\rho_{1}^{2}}}:=\rho_{2} \text {. }
$$

From (3.4), we have

$$
\begin{aligned}
\left|y^{\prime}\right|_{0} & \leq \max _{t \in[-k T, k T]}\left|f(x(t)) x^{\prime}(t)\right|+\max _{t \in[-k T, k T]}|g(x(t))|+\max _{t \in[-k T, k T]}\left|p_{k}(t)\right| \\
& \leq f_{\rho_{0}(\varepsilon)} \rho_{2}+g_{\rho_{0}(\varepsilon)}+|p|_{\infty} \\
& :=\rho_{3} .
\end{aligned}
$$

From (3.9)-(3.12), (3.17)-(3.19), we know that (3.2) and (3.3) are satisfied. Hence the conclusion of Theorem 3.1 holds.

Theorem 3.2 Suppose that conditions $\left(\mathrm{A}_{1}\right)$ and $\left(\mathrm{A}_{2}\right)$ hold. Furthermore, the positive constant $T$ satisfies (3.1). Then for each $k \in \mathbf{N},(2.2)$ has at least one $2 k T$-periodic solution $\left(x_{k}(t), y_{k}(t)\right)^{\top}$ in $\Sigma_{k} \subset X_{k}$ such that

$$
\begin{aligned}
& \left\|x_{k}\right\|_{m} \leq A_{0}, \quad\left\|x_{k}^{\prime}\right\|_{2} \leq A_{1}, \quad\left\|y_{k}\right\|_{2} \leq B_{0}, \quad\left\|y_{k}^{\prime}\right\|_{2} \leq B_{1}, \\
& \left|x_{k}\right|_{0} \leq \rho_{0}, \quad\left|y_{k}\right|_{0} \leq \rho_{1}<1, \quad\left|x_{k}^{\prime}\right|_{0} \leq \rho_{2}, \quad\left|y_{k}^{\prime}\right|_{0} \leq \rho_{3} \text {, }
\end{aligned}
$$

where $A_{0}, A_{1}, B_{0}, B_{1}, \rho_{0}, \rho_{1}, \rho_{2}, \rho_{3}$ are the constants defined in Theorem 3.1.

Proof In order to use Lemma 2.1, for each $k \in \mathbf{N}$, we consider the following system:

$$
\left\{\begin{array}{l}
x^{\prime}(t)=\lambda \varphi(y(t))=\lambda \frac{y(t)}{\sqrt{1-y^{2}(t)}} \\
y^{\prime}(t)=-\lambda f(x(t)) x^{\prime}(t)-\lambda g(x(t))+\lambda p_{k}(t), \quad \lambda \in(0,1),
\end{array}\right.
$$

a $2 k T$-periodic solution of system $(3.20)$. Since $(0,1) \subset(0,1]$, we have $\Omega_{1} \subset \Sigma_{k}$, where $\Sigma_{k}$ is defined in Theorem 3.1. If $(x, y)^{\top} \in \Omega_{1}$, by using Theorem 3.1, we get

$$
|x|_{0} \leq \rho_{0}, \quad|y|_{0} \leq \rho_{1}<1
$$


Let $\Omega_{2}=\left\{v=(x, y)^{\top} \in \operatorname{Ker} L, Q N v=0\right\}$. If $(x, y)^{\top} \in \Omega_{2}$, then $(x, y)^{\top}=\left(a_{1}, a_{2}\right)^{\top} \in R^{2}$ (constant vector), we see that

$$
\left\{\begin{array}{l}
a_{2}=0 \\
\int_{-k T}^{k T}\left[-g\left(a_{1}\right)+p_{k}(t)\right] d t=0
\end{array}\right.
$$

Multiplying the second equation of (3.21) by $a_{1}$, we have

$$
2 k \operatorname{Tm}_{0} a_{1}^{2} \leq \int_{-k T}^{k T} a_{1} p_{k}(t) d t \leq \sqrt{2 k T}\left|a_{1}\right| B
$$

thus

$$
\left|a_{1}\right| \leq \frac{B}{\sqrt{2 k T} m_{0}} \leq \frac{B}{\sqrt{2 T} m_{0}}:=\beta .
$$

Now, if we set $\Omega=\left\{v=(x, y)^{\top} \in X_{k},|x|_{0}<\rho_{0}+\beta,|y|_{0}<\rho^{*}<1\right\}$, where $\rho_{1}<\rho^{*}<1$, then $\Omega \supset \Omega_{1} \cup \Omega_{2}$. So condition (H1) and condition (H2) of Lemma 2.1 are satisfied. What remains is verifying condition (H3) of Lemma 2.1. In order to do this, let

$$
H(v, \mu):(\Omega \cap \operatorname{Ker} L) \times[0,1] \rightarrow R: \quad H(v, \mu)=\mu(x, y)^{\top}+(1-\mu) J Q N(v),
$$

where $J: \operatorname{Im} Q \rightarrow \operatorname{Ker} L$ is a linear isomorphism, $J(x, y)=(y, x)^{\top}$. From assumption $\left(\mathrm{A}_{1}\right)$, we have $v^{T} H(v, \mu) \neq 0, \forall(v, \mu) \in \partial \Omega \cap \operatorname{Ker} L \times[0,1]$. Hence

$$
\operatorname{deg}\{J Q N, \Omega \cap \operatorname{Ker} L, 0\}=\operatorname{deg}\{H(v, 0), \Omega \cap \operatorname{Ker} L, 0\}=\operatorname{deg}\{H(v, 1), \Omega \cap \operatorname{Ker} L, 0\} \neq 0 .
$$

So condition (H3) of Lemma 2.1 is satisfied. Therefore, by using Lemma 2.1, we see that (2.2) has a $2 k T$-periodic solution $\left(x_{k}, y_{k}\right)^{\top} \in \bar{\Omega}$. Obviously, $\left(x_{k}, y_{k}\right)^{\top}$ is a $2 k T$-periodic solution to (3.3) for the case of $\lambda=1$, so $\left(x_{k}, y_{k}\right)^{\top} \in \Sigma_{k}$. Thus, by using conclusions (3.2) and (3.3) in Theorem 3.1, we get

$$
\begin{aligned}
& \left\|x_{k}\right\|_{m} \leq A_{0}, \quad\left\|x_{k}^{\prime}\right\|_{2} \leq A_{1}, \quad\left\|y_{k}\right\|_{2} \leq B_{0}, \quad\left\|y_{k}^{\prime}\right\|_{2} \leq B_{1}, \\
& \left|x_{k}\right|_{0} \leq \rho_{0}, \quad\left|y_{k}\right|_{0} \leq \rho_{1}<1, \quad\left|x_{k}^{\prime}\right|_{0} \leq \rho_{2}, \quad\left|y_{k}^{\prime}\right|_{0} \leq \rho_{3} \text {. }
\end{aligned}
$$

Hence the conclusion of Theorem 3.2 holds. The proof is completed.

Theorem 3.3 Suppose that conditions $\left(\mathrm{A}_{1}\right)$ and $\left(\mathrm{A}_{2}\right)$ hold. Furthermore, the positive constant $T$ satisfies (3.1). Then (1.1) has at least one nontrivial homoclinic solution.

Proof By using Theorem 3.2, we see that, for each $k \in \mathbf{N}$, there exists a $2 k T$-periodic solution $\left(x_{k}, y_{k}\right)^{\top}$ to $(2.2)$ with

$$
\begin{aligned}
& \left\|x_{k}\right\|_{m} \leq A_{0}, \quad\left\|x_{k}^{\prime}\right\|_{2} \leq A_{1}, \quad\left\|y_{k}\right\|_{2} \leq B_{0}, \quad\left\|y_{k}^{\prime}\right\|_{2} \leq B_{1}, \\
& \left|x_{k}\right|_{0} \leq \rho_{0}, \quad\left|y_{k}\right|_{0} \leq \rho_{1}<1, \quad\left|x_{k}^{\prime}\right|_{0} \leq \rho_{2}, \quad\left|y_{k}^{\prime}\right|_{0} \leq \rho_{3} \text {, }
\end{aligned}
$$

where $A_{0}, A_{1}, B_{0}, B_{1}, \rho_{0}, \rho_{1}, \rho_{2}, \rho_{3}$ are constants independent of $k \in \mathbf{N}$. Equation (3.23) together with Lemma 2.3 shows that there is a function $u_{0}:=\left(x_{0}, y_{0}\right)^{\top} \in C\left(R, R^{2}\right)$ and a 
subsequence $\left\{\left(x_{k_{j}}, y_{k_{j}}\right)^{\top}\right\}$ of $\left\{\left(x_{k}, y_{k}\right)^{\top}\right\}_{k \in \mathbf{N}}$ such that for each interval $[a, b] \subset R, x_{k_{j}}(t) \rightarrow$ $x_{0}(t)$ and $y_{k_{j}}(t) \rightarrow y_{0}(t)$ uniformly on $[a, b]$. This together with the condition of $\left|y_{k}\right|_{0} \leq$ $\rho_{1}<1$ in (3.23) implies that

$$
\max _{t \in[a, b]}\left|y_{0}(t)\right| \leq \rho_{1}<1 \quad \text { for any } a, b \in R \text { with } a<b
$$

Below, we will show that $\left(x_{0}(t), y_{0}(t)\right)^{\top}$ is just a homoclinic solution to (1.1).

Since $\left(x_{k}(t), y_{k}(t)\right)^{\top}$ is a $2 k T$-periodic solution of (2.2), it follows that

$$
\left\{\begin{array}{l}
x_{k}^{\prime}(t)=\varphi\left(y_{k}(t)\right)=\frac{y_{k}(t)}{\sqrt{1-y_{k}^{2}(t)}}, \\
y_{k}^{\prime}(t)=-f\left(x_{k}(t)\right) \varphi\left(y_{k}(t)\right)-g\left(x_{k}(t)\right)+p_{k}(t) .
\end{array}\right.
$$

For all $a, b \in R$ with $a<b$, there must be a positive integer $j_{0}$ such that for $j>j_{0}$, $\left[-k_{j} T, k_{j} T-\varepsilon_{0}\right] \supset[a, b]$. So for $j>j_{0}$, from (1.4) and (3.25) we see that

$$
\left\{\begin{array}{l}
x_{k_{j}}^{\prime}(t)=\varphi\left(y_{k_{j}}(t)\right)=\frac{y_{k_{j}}(t)}{\sqrt{1-y_{k_{j}}^{2}(t)}}, \\
y_{k_{j}}^{\prime}(t)=-f\left(x_{k_{j}}(t)\right) \varphi\left(y_{k_{j}}(t)\right)-g\left(x_{k_{j}}(t)\right)+p(t), \quad t \in(a, b),
\end{array}\right.
$$

which together with (3.24) results in

$$
x_{k_{j}}^{\prime}(t)=\frac{y_{k_{j}}(t)}{\sqrt{1-y_{k_{j}}^{2}(t)}} \rightarrow \frac{y_{0}(t)}{\sqrt{1-y_{0}^{2}(t)}}
$$

and

$$
\begin{aligned}
y_{k_{j}}^{\prime}(t) & =-f\left(x_{k_{j}}(t)\right) \varphi\left(y_{k_{j}}(t)\right)-g\left(x_{k_{j}}(t)\right)+p(t) \\
& \rightarrow-f\left(x_{0}(t)\right) \frac{y_{0}(t)}{\sqrt{1-y_{0}^{2}(t)}}-g\left(x_{0}(t)\right)+p(t)
\end{aligned}
$$

uniformly for $t \in[a, b]$ as $j \rightarrow+\infty$. Since $x_{k_{j}}(t) \rightarrow x_{0}(t)$ and $x_{k_{j}}(t)$ is continuously differentiable for $t \in(a, b)$, it follows that

$$
x_{k_{j}}^{\prime}(t) \rightarrow x_{0}^{\prime}(t) \text { uniformly for } t \in[a, b] \text { as } j \rightarrow+\infty,
$$

which together with (3.26) yields

$$
x_{0}^{\prime}(t)=\frac{y_{0}(t)}{\sqrt{1-y_{0}^{2}(t)}}, \quad t \in(a, b) .
$$

Similarly, by (3.27) we have

$$
y_{0}^{\prime}(t)=-f\left(x_{0}(t)\right) \frac{y_{0}(t)}{\sqrt{1-y_{0}^{2}(t)}}-g\left(x_{0}(t)\right)+p(t), \quad t \in(a, b) .
$$


Considering $a, b$ are two arbitrary constants with $a<b$, it is easy to see that $\left(x_{0}(t), y_{0}(t)\right)^{\top}$, $t \in R$, is a solution to (2.2), i.e.,

$$
\left\{\begin{array}{l}
x_{0}^{\prime}(t)=\frac{y_{0}(t)}{\sqrt{1-y_{0}^{2}(t)}} \\
y_{0}^{\prime}(t)=-f\left(x_{0}(t)\right) \frac{y_{0}(t)}{\sqrt{1-y_{0}^{2}(t)}}-g\left(x_{0}(t)\right)+p(t) .
\end{array}\right.
$$

Now, we will prove $x_{0}(t) \rightarrow 0$ and $x_{0}^{\prime}(t) \rightarrow 0$ as $|t| \rightarrow+\infty$.

Since

$$
\begin{aligned}
\int_{-\infty}^{+\infty}\left(\left|x_{0}(t)\right|^{m}+\left|x_{0}^{\prime}(t)\right|^{2}\right) d t & =\lim _{i \rightarrow+\infty} \int_{-i T}^{i T}\left(\left|x_{0}(t)\right|^{m}+\left|x_{0}^{\prime}(t)\right|^{2}\right) d t \\
& =\lim _{i \rightarrow+\infty} \lim _{j \rightarrow+\infty} \int_{-i T}^{i T}\left(\left|x_{k_{j}}(t)\right|^{m}+\left|x_{k_{j}}^{\prime}(t)\right|^{2}\right) d t
\end{aligned}
$$

clearly, for every $i \in \mathbf{N}$, if $k_{j}>i$, by (3.22),

$$
\int_{-i T}^{i T}\left(\left|x_{k_{j}}(t)\right|^{m}+\left|x_{k_{j}}^{\prime}(t)\right|^{2}\right) d t \leq \int_{-k_{j} T}^{k_{j} T}\left(\left|x_{k_{j}}(t)\right|^{m}+\left|x_{k_{j}}^{\prime}(t)\right|^{2}\right) d t \leq A_{0}^{m}+A_{1}^{2} .
$$

Let $i \rightarrow+\infty$ and $j \rightarrow+\infty$. We have

$$
\int_{-\infty}^{+\infty}\left(\left|x_{0}(t)\right|^{m}+\left|x_{0}^{\prime}(t)\right|^{2}\right) d t \leq A_{0}^{2}+A_{1}^{2}
$$

and then

$$
\int_{|t| \geq r}\left(\left|x_{0}(t)\right|^{m}+\left|x_{0}^{\prime}(t)\right|^{2}\right) d t \rightarrow 0
$$

as $r \rightarrow+\infty$. So by using Lemma 2.2, we obtain

$$
\begin{aligned}
\left|x_{0}(t)\right| & \leq(2 T)^{-\frac{1}{m}}\left(\int_{t-T}^{t+T}|x(s)|^{m} d s\right)^{\frac{1}{m}}+T(2 T)^{-\frac{1}{2}}\left(\int_{t-T}^{t+T}\left|x^{\prime}(s)\right|^{2} d s\right)^{\frac{1}{2}} \\
& \leq\left[(2 T)^{-\frac{1}{m}}+T(2 T)^{-\frac{1}{2}}\right]\left[\left(\int_{t-T}^{t+T}|x(s)|^{m} d s\right)^{1 / m}+\left(\int_{t-T}^{t+T}\left|x^{\prime}(s)\right|^{2} d s\right)^{\frac{1}{2}}\right] \\
& \rightarrow 0 \quad \text { as }|t| \rightarrow+\infty,
\end{aligned}
$$

which implies that

$$
x_{0}(t) \rightarrow 0 \quad \text { as }|t| \rightarrow+\infty .
$$

Similarly, we can prove that

$$
y_{0}(t) \rightarrow 0 \quad \text { as }|t| \rightarrow+\infty
$$

which together with the first equation of (3.28) gives

$$
x_{0}^{\prime}(t) \rightarrow 0 \quad \text { as }|t| \rightarrow+\infty .
$$


It is easy to see from (3.28) that $x_{0}(t)$ is a solution for (1.1). Thus, by (3.29) and (3.30), $x_{0}(t)$ is just a homoclinic solution to (1.1). Clearly, $x_{0}(t) \not \equiv 0$, otherwise, $p(t) \equiv 0$, which contradicts assumption $\left(\mathrm{A}_{2}\right)$. Hence the conclusion of Theorem 3.3 holds. The proof is completed.

Theorem 3.4 Suppose that $x g(x) \leq 0$ for $x \in(-\infty, 0) \cup(0,+\infty)$ and $u_{0}(t)$ is an arbitrary homoclinic solution to (1.1). Then the following statements are true:

(1) If $p(t) \geq 0(p(t)>0)$ for all $t \in R$, then $u_{0}(t) \leq 0\left(u_{0}(t)<0\right)$ for all $t \in R$.

(2) If $p(t) \leq 0(p(t)<0)$ for all $t \in R$, then $u_{0}(t) \geq 0\left(u_{0}(t)<0\right)$ for all $t \in R$.

Proof We only prove the cases outside the brackets.

(1) Suppose that $u_{0}(t)$ is an arbitrary homoclinic solution to (1.1), then

$$
\left(\frac{u_{0}^{\prime}(t)}{\sqrt{1+\left(u_{0}^{\prime}(t)\right)^{2}}}\right)^{\prime}+f\left(u_{0}(t)\right) u_{0}^{\prime}(t)+g\left(u_{0}(t)\right)=p(t)
$$

and

$$
u_{0}(t) \rightarrow 0, \quad u_{0}^{\prime}(t) \rightarrow 0 \quad \text { as }|t| \rightarrow+\infty .
$$

If statement (1) in Theorem 3.4 is not true, there must be a point $t^{*} \in R$ such that $u_{0}\left(t^{*}\right)>0$. It follows from (3.32) that there is a constant $\rho>0$ such that $t^{*} \in(-\rho, \rho)$ and $u_{0}(t)<\frac{u\left(t^{*}\right)}{2}$ for $t \in(-\infty, \rho) \cup(\rho,+\infty)$. Let $t^{* *} \in[-\rho, \rho]$ such that $u_{0}\left(t^{* *}\right)=\max _{t \in[-\rho, \rho]} u(t)$, then

$$
u_{0}\left(t^{* *}\right) \geq u_{0}\left(t^{*}\right)>0
$$

and

$$
u_{0}\left(t^{* *}\right) \geq u_{0}\left(t^{*}\right)>\sup _{t \in(-\infty, \rho) \cup(\rho,+\infty)} u_{0}(t)
$$

i.e.,

$$
u_{0}\left(t^{* *}\right)=\max _{t \in R} u_{0}(t)
$$

So $u_{0}^{\prime}\left(t^{* *}\right)=0$ and $\left.\left(\frac{u_{0}^{\prime}(t)}{\sqrt{1+\left(u_{0}^{\prime}(t)\right)^{2}}}\right)^{\prime}\right|_{t=t^{* *}} \leq 0$, and then from (3.31) we see

$$
g\left(u_{0}\left(t^{* *}\right)\right)=-\left.\left(\frac{u_{0}^{\prime}(t)}{\sqrt{1+\left(u_{0}^{\prime}(t)\right)^{2}}}\right)^{\prime}\right|_{t=t^{* *}}+p\left(t^{* *}\right)
$$

i.e.,

$$
g\left(u_{0}\left(t^{* *}\right)\right) \geq 0 .
$$

By using the assumption of $x g(x) \leq 0$ for $x \in(-\infty, 0) \cup(0,+\infty)$, we have $u_{0}\left(t^{* *}\right) \leq 0$, which contradicts (3.33). This contradiction implies that statement (1) in Theorem 3.4 is true. 
Similarly, we can prove that statement (2) in Theorem 3.4 is also true. The proof is completed.

By using Theorem 3.4, we can obtain the following results.

Corollary 3.1 Suppose that $x g(x) \leq 0$ for $x \in(-\infty, 0) \cup(0,+\infty)$. If $p(t) \equiv 0$, then $(1.1)$ has no nontrivial homoclinic solution.

Corollary 3.2 Suppose that $x g(x) \leq 0$ for $x \in(-\infty, 0) \cup(0,+\infty)$ and $u_{0}(t)$ is a homoclinic solution to (1.1). If there are two points $t_{1}, t_{2} \in R$ such that $u_{0}\left(t_{1}\right) u_{0}\left(t_{2}\right)<0$, then there must be two points $t_{3}, t_{4} \in R$ such that $p\left(t_{3}\right) p\left(t_{4}\right)<0$.

As an application, we consider the following example:

$$
\left(\frac{u^{\prime}(t)}{\sqrt{1+\left(u^{\prime}(t)\right)^{2}}}\right)^{\prime}-\left(1+\frac{u^{2}(t)}{1++u^{2}(t)}\right) u^{\prime}(t)-3(u(t))^{3}=\frac{\theta e^{t / 2}}{e^{-t}+e^{t}},
$$

where $\theta \in R$ is a constant. Corresponding to (1.1), we can chose $p(t)=\frac{\theta e^{t / 2}}{e^{-t}+e^{t}}, m=4, m_{0}=3$, and $l=1$ such that assumption $\left(\mathrm{A}_{1}\right)$ holds. Furthermore, by a direct calculation, we can easily obtain

$$
\int_{-\infty}^{+\infty}|p(t)|^{2} d t=\frac{\theta^{2} \pi}{4}<\infty, \quad \int_{R}|p(t)|^{\frac{m}{m-1}} d t=\int_{-\infty}^{+\infty}|p(t)|^{\frac{4}{3}} d t=\frac{3}{2} \theta^{4 / 3}<\infty .
$$

This implies that assumption $\left(\mathrm{A}_{2}\right)$ also holds, and $\sup _{t \in R}|p(t)|=\frac{\theta}{4} 3^{\frac{3}{4}}<\frac{3 \theta}{4}, \alpha=\left(\frac{3}{2}\right)^{\frac{3}{4}} \theta, \beta=$ $\frac{2}{\sqrt{\pi}} \theta$. Let $T=\frac{1}{2}$. Since $\lim _{|\theta| \rightarrow+\infty} \rho_{1}=+\infty$ and $\lim _{\theta \rightarrow 0} \rho_{1}=0$, where $\rho_{1}=(2 T)^{-\frac{1}{2}}\left(\frac{\alpha^{m}}{m_{0}}\right)^{\frac{1}{2(m-1)}}+$ $\beta \sqrt{\frac{T}{2}}\left[\frac{M}{l}+1\right]$ is determined in (3.1), it follows that there is constant $\theta_{0}>0$ such that

$$
\rho_{1}=(2 T)^{-\frac{1}{2}}\left(\frac{\alpha^{m}}{m_{0}}\right)^{\frac{1}{2(m-1)}}+\beta \sqrt{\frac{T}{2}}\left[\frac{M}{l}+1\right]<1, \quad \forall \theta \in\left(-\theta_{0}, 0\right) \cup\left(0, \theta_{0}\right) .
$$

So by applying Theorem 3.3, Theorem 3.4, and Corollary 3.1, we can obtain the following results:

(1) If $\theta \in\left(-\theta_{0}, 0\right) \cup\left(0, \theta_{0}\right)$, then (3.34) has at least one nontrivial homoclinic solution.

Furthermore, if $\theta \in\left(-\theta_{0}, 0\right)$, then all the homoclinic solutions to (3.34) are positive; if $\theta \in\left(0, \theta_{0}\right)$, then all the homoclinic solutions to (3.34) are negative.

(2) If $\theta=0$, then (3.34) has no nontrivial homoclinic solution.

\section{Competing interests}

The author declares that they have no competing interests.

\section{Acknowledgements}

Research was supported by the NNSF of China (No. 11271197). The author is very grateful to the referee for her/his careful reading of the original manuscript and for her/his valuable suggestions for improving this article.

Received: 1 February 2015 Accepted: 18 July 2015 Published online: 04 August 2015

\section{References}

1. Bergner, M: On the Dirichlet problem for the prescribed mean curvature equation over general domains. Differ. Geom. Appl. 27, 335-343 (2009)

2. Rey, O: Heat flow for the equation of surfaces with prescribed mean curvature. Math. Ann. 297, 123-146 (1991) 
3. Amster, P, Mariani, MC: The prescribed mean curvature equation for nonparametric surfaces. Nonlinear Anal. 52, 1069-1077 (2003)

4. Finn, R: Equilibrium Capillary Surfaces. Springer, New York (1986)

5. Kurganov, A, Rosenau, P: On reaction processes with saturating diffusion. Nonlinearity 19, 171-193 (2006)

6. Giusti, E: Minimal Surfaces and Functions of Bounded Variations. Birkhäuser, Basel (1984)

7. Pan, $\mathrm{H}$ : One-dimensional prescribed mean curvature equation with exponential nonlinearity. Nonlinear Anal. 70, 999-1010 (2009)

8. Obersnel, F, Omari, P: Positive solutions of the Dirichlet problem for the prescribed mean curvature equation. J. Differ. Equ. 249, 1674-1725 (2010)

9. Benevieri, P, Doó, JM, De Medeiros, ES: Periodic solutions for nonlinear equations with mean curvature-like operators. Appl. Math. Lett. 20, 484-492 (2007)

10. Benevieri, P, Doó, JM, De Medeiros, ES: Periodic solutions for nonlinear systems with mean curvature-like operators. Nonlinear Anal. 65, 1462-1475 (2006)

11. Feng, M: Periodic solutions for prescribed mean curvature Liénard equation with a deviating argument. Nonlinear Anal., Real World Appl. 13, 1216-1223 (2012)

12. Lu, S, Lu, M: Periodic solutions for a prescribed mean curvature equation with multiple delays. J. Appl. Math. 2014 Article ID 909252 (2014). doi:10.1155/2014/909252

13. Li, J: Periodic solutions for prescribed mean curvature Rayleigh equation with a deviating argument. Adv. Differ. Equ. 2013, 88 (2013)

14. Rabinowitz, PH: Homoclinic orbits for a class of Hamiltonian systems. Proc. R. Soc. Edinb., Sect. A 114, 33-38 (1990)

15. Izydorek, M, Janczewska, J: Homoclinic solutions for a class of the second order Hamiltonian systems. J. Differ. Equ. 219, 375-389 (2005)

16. Tang, $\mathrm{XH}, \mathrm{XiaO}, \mathrm{L}$ : Homoclinic solutions for ordinary $p$-Laplacian systems with a coercive potential. Nonlinear Anal. 71, 1124-1132 (2009)

17. Izydorek, M, Janczewska, J: Homoclinic solutions for nonautonomous second-order Hamiltonian systems with a coercive potential. J. Math. Anal. Appl. 335, 1119-1127 (2007)

18. Liang, Z, Lu, S: Homoclinic solutions for a kind of prescribed mean curvature Duffing-type equation. Adv. Differ. Equ. 2013, 279 (2013). http://www.advancesindifferenceequations.com/content/2013/1/279

19. Lu, S: Homoclinic solutions for a class of second-order $p$-Laplacian differential systems with delay. Nonlinear Anal. Real World Appl. 12, 780-788 (2011)

20. Gaines, RE, Mawhin, JL: Coincidence Degree and Nonlinear Differential Equation. Springer, Berlin (1977)

21. Lu, S: Existence of homoclinic solutions for a class of neutral functional differential equations. Acta Math. Sin. Engl. Ser. 28, 1261-1274 (2012)

\section{Submit your manuscript to a SpringerOpen ${ }^{\ominus}$ journal and benefit from:}

$\checkmark$ Convenient online submission

Rigorous peer review

- Immediate publication on acceptance

Open access: articles freely available online

High visibility within the field

- Retaining the copyright to your article 